\title{
Critical Assessment of Noninvasive Prenatal Testing: Before or after Anomaly Scan
}

Yaron Zalel

How to cite this article: Zalel Y. Critical Assessment of Noninvasive Prenatal Testing: Before or after Anomaly Scan. Donald School J Ultrasound Obstet Gynecol 2018;12(4):255-257.

Source of support: Nil

\section{Conflict of interest: None}

Noninvasive prenatal test (NIPT) analyzes cell-free DNA (cfDNA) in the maternal serum. Approximately, $3-15 \%$ of cfDNA in the maternal blood is of fetal origin.

The NIPT examination, based on the existence of this cfDNA, is accurate in screening for Down syndrome and less for other trisomies, as well as other structural chromosomal abnormalities. The commercial companies offer this technique as early as 10 weeks of gestation.

The purpose of this critical assessment is to challenge the timing of offering the NIPT to women at 10 weeks gestation, emphasizing the limitations of this technique before the anomaly scan is done.

The utilization of NIPT for genetic screening has increased rapidly since the introduction of the first clinical test in October 2011. However, its performance early in pregnancy hasnot been recommended by the commercial companies only. Likewise, Bianchi et al. ${ }^{1}$ have even examined their patients from 8 weeks gestation, Lo et al. ${ }^{2}$ stated that it can be done after 9-10 weeks' gestation and the Israeli Society of Medical Genetics NIPT Committee Opinion $072013^{3}$ stated that it can be done as early as 10 weeks of gestation.

Furthermore, although the 99\% detection rate for trisomy 21, using cfDNA screening, one should remember, that cfDNA analysis is a screening test and is not considered diagnostic because of the potential for falsepositive results. Positive results require secondary testing with invasive techniques.

Moreover, $17 \%$ of chromosomal abnormalities detected by a traditional screening test were not detectable by cfDNA screening. ${ }^{4,5}$ These abnormalities included rare trisomies, unbalanced rearrangements, and large deletions or duplications.

Professor

Expert Clinic, Habarzel 9a Tel-Aviv, Israel

Corresponding Author: Yaron Zalel, Professor, Expert Clinic, Habarzel 9a Tel-Aviv, Israel, Phone: 972-52-6666462, e-mail: zalel1954@gmail.com
I have published in 2015 an article named: NIPT-it is all a matter of timing. ${ }^{6}$ I have described, during the first year after the introduction of the technique to Israel, ${ }^{6}$ women with various malformations (including severe heart, brain, and renal abnormalities) found in anomaly scan a few days after a normal NIPT. These women were falsely reassured of the "normality" of their pregnancies, eventually either underwent a further invasive procedure or terminated the pregnancy and even paid considerable sums of money for the blood test.

While preparing this opinion, I have turned to G-Med raising the same issue.

Global Online Medical Community (G-Med) is the largest community for physicians, true medical crowdsourcing enabled by peer-to-peer consultation of hundred thousands of physicians worldwide (www.g-med.info).

I have added a recent case of mine (a 41 years old woman, para 0 , after normal NIPT examination at 10 weeks gestation, who was diagnosed with multiple anomalies, including a large omphalocele containing liver, complex heart disease and IUGR (Figs 1 to 4 ) found at early anomaly scan that led to a termination of pregnancy) and raised the question of timing of the NIPT.

These were the results:
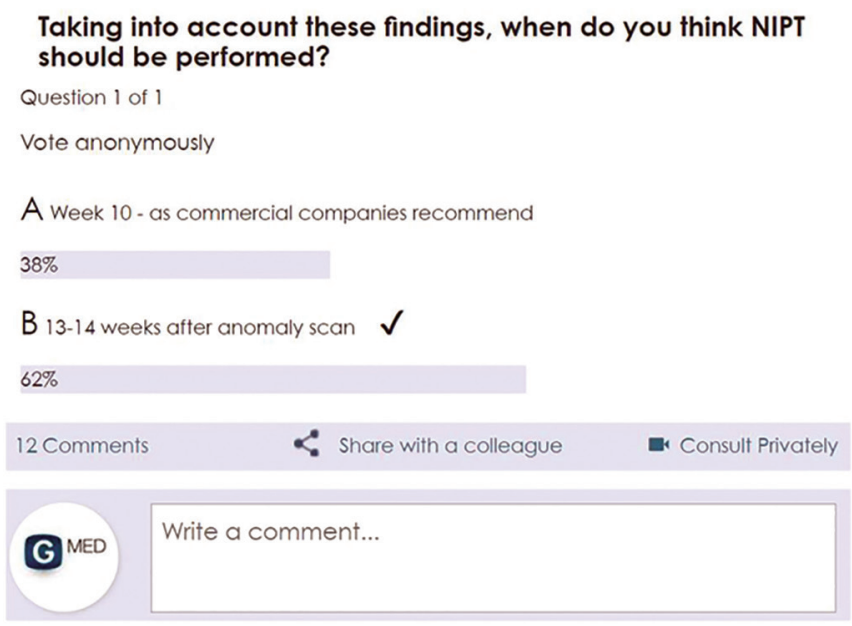

So, although we have succeeded to raise the importance of performing an anomaly scan before the NIPT, there are still $38 \%$ of the physicians worldwide that would send their patients for NIPT first.

Furthermore, in various countries, including the US, there are many NIPT examinations done 


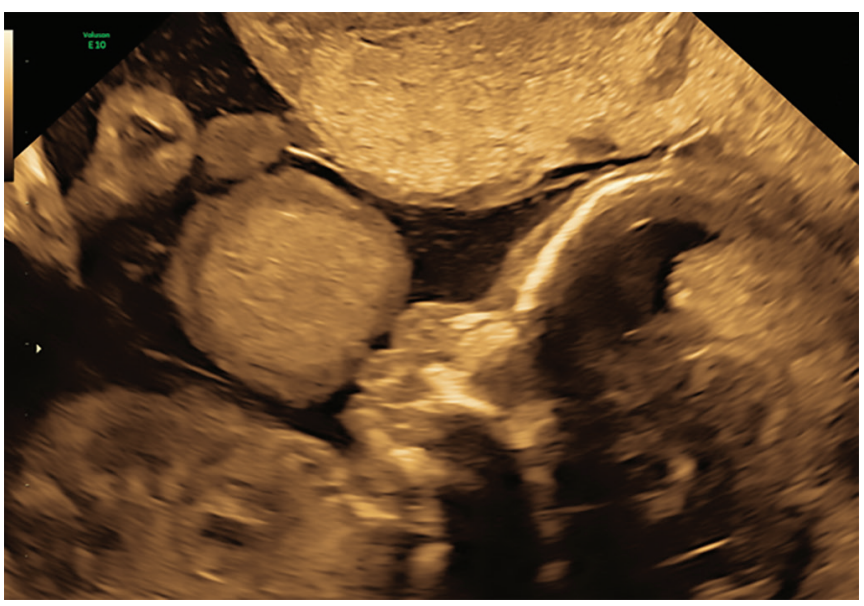

Fig. 1: The fetus "watches" large omphalocele in front of its face (2D)

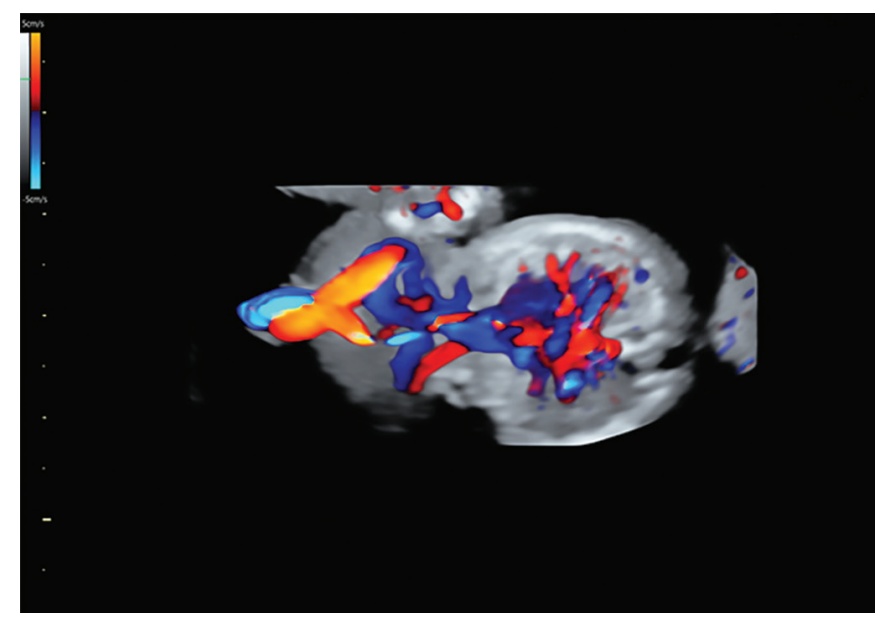

Fig. 3: Fetal abdomen (axial view, 3D color Doppler)—Blood vessels within the omphalocele

primarily in general OB offices, leading to a suboptimal discussion regarding limitations and benefits with the patient. The problem is, however, much more serious than that. The false-reassurance given to these low-risk patients after a normal NIPT for 9-10 weeks gestation, leads in many cases to eliminate the Nuchal scan at 11-13 weeks or even the early anomaly scan for $14-16$ weeks due to inappropriate counseling regarding the importance of an anatomic evaluation than just "A Down syndrome test".

This technique has its obvious advantages-the ability to get quite accurate results of the fetal DNA, especially regarding trisomy 21 and so early in pregnancy. Nevertheless, current guidelines from the American College of Medical Genetics, the American College of Obstetricians and Gynecologists, and the Society for Maternal Fetal Medicine advise that cfDNA may be offered to all women to screen for common aneuploidies and fetal sex, but it is not considered the standard approach in pregnancies that are not considered high risk. $^{7}$

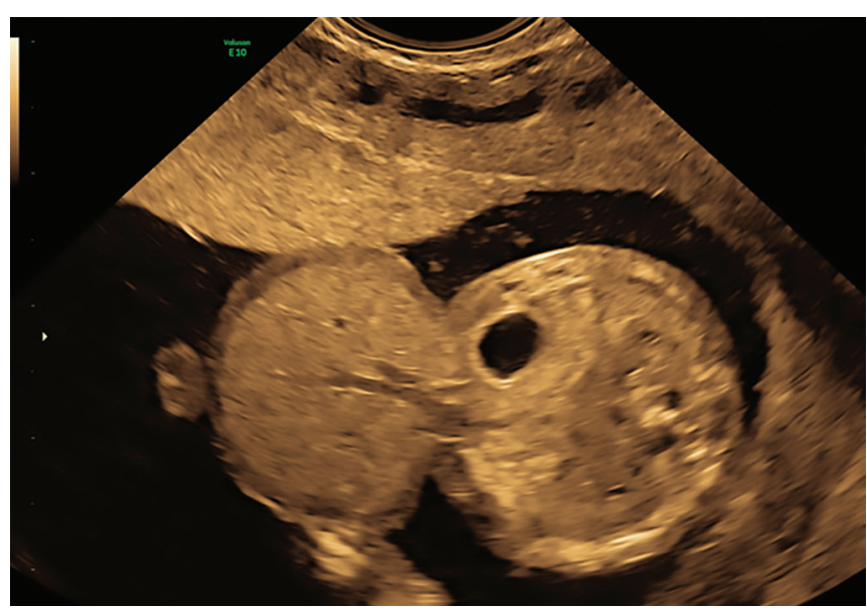

Fig. 2: Large omphalocele containing liver (2D)

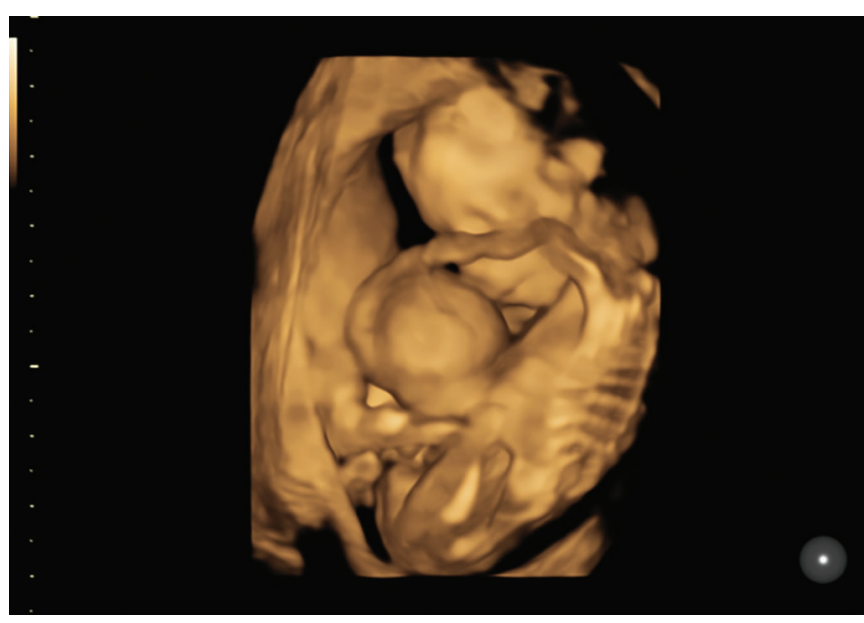

Fig. 4: Whole fetus (3D) with large omphalocele

Lately, Allyse and Wick, ${ }^{8}$ at the JAMA clinical update, have stated that cfDNA screening can detect chromosome aneuploidy in pregnancy after 10 weeks' gestation but is less effective at screening for other genetic abnormalities. They also added that cfDNA tests are more expensive than other approaches.

When we recommend an early anatomy scan done before the NIPT examination, we have to ask are we capable of performing an anatomy scan so early in pregnancy?

In most of the cardinal chromosomal syndromes: trisomy $21,13,18, X_{0}$, triploidy-there is a severe structural anomaly, especially cardiac abnormality.

Concerning congenital heart diseases (CHD), Yagel et al. ${ }^{9}$ have found that $64 \%$ of $\mathrm{CHD}$ are detectable during the first TVS at $13-16$ weeks, and Moyano et al. ${ }^{10}$ have stated that diagnosis of heart malformations can be made reliably, even in the first trimester at the time of nuchal translucency measurement.

Bronshtein et al. ${ }^{11}$ have found while evaluating fetuses with increased nuchal translucency (NT) at 
an early (11-14 gestational weeks), that overall, there were $27 \%$ fetuses with severe structural anomalies. The remaining 73\% underwent CVS which was found abnormal in $33 \%$.

Zalel et al. ${ }^{12,13}$ share the same experience. We have examined 32 patients with a mean gestational age at a scan of 12.3 weeks (11-14 weeks) with mean NT of $4.6 \mathrm{~mm}$ (range 3.4-9). Overall, our study has found major abnormalities in $78 \%$ of the cases. The presence of malformations yielded a positive predictive value of $53 \%,{ }^{8}$ and $96.7 \%$ for having abnormal karyotype or abnormal outcome, respectively. The absence of malformations yielded a negative predictive value of $92.3 \%$ and $84.6 \%$, respectively. We have summarized that early (11-14 weeks) First-trimester fetal anomaly scan, including echocardiography is feasible and accurate.

Therefore, Benn et al., 14 have adopted the "contingent approach"-since universal NIPT is not cost-effective, performing NIPT only contingently in women found at moderate or high risk by conventional screening is cost-effective. We strongly agree with this statement and suggest not to lean on NT scan alone, but to do the NIPT only after an early anomaly scan is done. Another advantage of karyotyping the fetus only after anomaly scan is done to recommend doing also microarray testing in cases where the abnormality is found instead of the NIPT or the normal karyotype (as an evaluation for VCF in cases of heart malformations).

\section{SUMMARY}

Given the above data, I challenge the timing of offering the NIPT to all women at 10 weeks gestation. I suggest a re-evaluation of my previous suggestion-a different contingent approach not based solely on NT measurement but to recommend all women and their physicians of postponing NIPT only until an early anomaly scan is performed at around 13-14 weeks of gestation (or even earlier in cases of increased NT).

It is true that this approach would delay NIPT results until after the anomaly scan. However, there is little difference between termination of pregnancy at 12 weeks and 14 weeks of gestation. Moreover, this will save the money spent on this test, will spare the patient "false reassurance" of the normality of the pregnancy, and in cases where major abnormalities were excluded, it will allow further evaluation of the pregnancy either by NIPT or invasive karyotyping. This approach will also eliminate (hopefully) the trend towards performing the NIPT alone while avoiding additional important information gained by the structural anomaly scan and thus turning the pre-natal care to "Down syndrome test alone".

Author thinks this message should be communicated to the commercial companies as well as to the physician that consult their patients regarding the timing of the NIPT technique.

\section{REFERENCES}

1. Bianchi DW, Parker RL, Wentworth J, et al. DNA sequencing versus standard prenatal aneuploidy screening. New England journal of medicine. $2014 \mathrm{Feb}$ 27;370(9):799-808.

2. Lo JO, Cori D F, Norton ME, et al. Noninvasive prenatal testing. Obstet Gynecol Surv. 2014 Feb;69(2):89-99.

3. Michaelson-Cohen R, Gershoni-Baruch R, Sharoni R, et al. Israeli society of medical genetics NIPT committee opinion 072013: non-invasive prenatal testing of cell-free DNA in maternal plasma for detection of fetal aneuploidy. Fetal diagnosis and therapy. 2014;36(3):242-244.

4. Oepkes D, Tabor A, Yaron Y. Prenatal aneuploidy screening using cell free DNA. Am J Obstet Gynecol. 2015;213(4):596597.

5. Norton ME, Jelliffe-Pawlowski LL, Currier RJ. Chromosome abnormalities detected by current prenatal screening and noninvasive prenatal testing. Obstet Gynecol. 2014;124(5): 979-986.

6. Zalel Y. Non-invasive prenatal testing--it's all a matter of timing. Ultrasound Obstet Gynecol. 2015 Jan;45(1):115-116

7. Gregg AR, Skotko BG, Benkendorf JL, et al. Noninvasive prenatal screening for fetal aneuploidy, 2016 update. Genet Med. 2016;18(10):1056-1065.

8. Allyse MA, Wick MJ. Noninvasive prenatal genetic screening using cell-free DNA. Jama. 2018 Aug 14;320(6):591-592.

9. Yagel S, Weissman A, Rotstein Z, et al. Congenital heart defects: natural course and in utero development. Circulation. 1997 Jul 15;96(2):550-555.

10. Moyano D, Huggon IC, Allan LD. Fetal echocardiography in trisomy 18. Arch Dis Child Fetal Neonatal Ed 2005;90:F520-F522.

11. Bronshtein M, Zimmer EZ, Blazer S. The Utility of Detailed First Trimester Ultrasound Examination Performed upon Detection of Abnormal Fetal Nuchal translucency. Prenat Diagn. 2008 Nov;28(11):1037-1041.

12. Zalel Y. Increased NT- to scan or to karyotype? P01.24. 24th World Congress on ultrasound in Obstetrics and Gynecology, Barcelona, Spain, September 2014.

13. Zalel Y, Zemet R, Kivilevitch Z. The added value of detailed early anomaly scan in fetuses with increased nuchal translucency. PrenatDiagn. 2017 Mar;37(3):235-243.

14. Benn P, Cuckle H, Pergament E. Non-invasive prenatal testing for aneuploidy: current status and future prospects. Ultrasound Obstet Gynecol. 2013 Jul;42(1):15-33. 